DOI: 10.17516/1997-1397-2020-13-3-331-341

УДК 519.21

\title{
On Construction of Positive Closed Currents with Prescribed Lelong Numbers
}

\author{
Hedi Khedhiri* \\ University of Monastir \\ Monastir, Tunisia
}

Received 06.01.2020, received in revised form 06.02.2020, accepted 09.03.2020

\begin{abstract}
We establish that a sequence $\left(X_{k}\right)_{k \in \mathbb{N}}$ of analytic subsets of a domain $\Omega$ in $\mathbb{C}^{n}$, purely dimensioned, can be released as the family of upper-level sets for the Lelong numbers of some positive closed current. This holds whenever the sequence $\left(X_{k}\right)_{k \in \mathbb{N}}$ satisfies, for any compact subset $L$ of $\Omega$, the growth condition $\sum_{k \in \mathbb{N}} C_{k} \operatorname{mes}\left(X_{k} \cap L\right)<\infty$. More precisely, we built a positive closed current $\Theta$ of bidimension $(p, p)$ on $\Omega$, such that the generic Lelong number $m_{X_{k}}$ of $\Theta$ along each $X_{k}$ satisfies $m_{X_{k}}=C_{k}$. In particular, we prove the existence of a plurisubharmonic function $v$ on $\Omega$ such that, each $X_{k}$ is contained in the upper-level set $E_{C_{k}}\left(d d^{c} v\right)$.

Keywords: closed positive current, plurisubharmonic function, potential, analytic set, Lelong number. Citation: H.Khedhiri, On Construction of Positive Closed Currents with Prescribed Lelong Numbers, J. Sib. Fed. Univ. Math. Phys., 2020, 13(3), 331-341.

DOI: $10.17516 / 1997-1397-2020-13-3-331-341$.
\end{abstract}

\section{Introduction}

We consider respectively, a domain $\Omega$ in $\mathbb{C}^{n}$, a fixed integer $1 \leqslant p \leqslant n$, a sequence of positive real numbers $\left(C_{k}\right)_{k \in \mathbb{N}}$ and a sequence $\left(X_{k}\right)_{k \in \mathbb{N}}$ of analytic subsets of $\Omega$, purely dimensioned such that

$$
\text { for all } k \in \mathbb{N}, \quad \operatorname{Codim}\left(X_{k}\right)=n-d_{k} \geqslant n-p \text {. }
$$

We study the existence of a closed positive current $\Theta$, such that for all $k \in \mathbb{N}$, the generic Lelong number of $\Theta$ along $X_{k}$ satisfies $m_{X_{k}}=C_{k}$. The existence of a solution will be shown under an appropriate growth condition on the family $\left(X_{k}\right)_{k}$ of the form

$$
\sum_{k \in \mathbb{N}} C_{k} \operatorname{mes}\left(X_{k} \cap L\right)=\sum_{k \in \mathbb{N}} C_{k} \int_{L}\left[X_{k}\right] \wedge \beta^{d_{k}}<\infty,
$$

for any compact subset $L \subset \Omega$.

With the above data, we state the main result of the paper as follows:

Theorem 0.1. Let $\left(C_{k}\right)_{k \in \mathbb{N}}$ be a sequence of positive real numbers and $\left(X_{k}\right)_{k \in \mathbb{N}}$ be a sequence of analytic subsets, purely dimensioned in a domain $\Omega$ of $\mathbb{C}^{n}$. Assume we have for any open ball $B(a, r) \Subset \Omega$ the condition $\sum_{k \in \mathbb{N}} C_{k} m e s\left(X_{k} \cap B(a, r)\right)<\infty$. Then there exists a positive closed $(n-p, n-p)$-current $\Theta$ on $\Omega$, such that for all $k \in \mathbb{N}, X_{k} \subset E_{C_{k}}(\Theta)$.

In particular, for plurisubharmonic functions on $\Omega$, we prove the following similar result:

*khediri_h@yahoo.fr

(C) Siberian Federal University. All rights reserved 
Theorem 0.2. Let $\left(C_{k}\right)_{k \in \mathbb{N}}$ be a sequence of positive real numbers and $\left(X_{k}\right)_{k \in \mathbb{N}}$ be a sequence of analytic subsets purely dimensioned in a domain $\Omega$ of $\mathbb{C}^{n}$. Assume we have for any open ball $B(a, r) \Subset \Omega$ the condition $\sum_{k \in \mathbb{N}} C_{k} \operatorname{mes}\left(X_{k} \cap B(a, r)\right)<\infty$. Then there exists a plurisubharmonic function $v$ on $\Omega$, such that for all $k \in \mathbb{N}, X_{k} \subset E_{C_{k}}\left(d d^{c} v\right)$.

\section{Problem statement and description of the objective of the paper}

The study presented in this paper is motivated by J.-P. Demailly during a visit of the author to the Fourier Institute.

Let $1 \leqslant p \leqslant n$ be a fixed natural number and $\left(X_{k}\right)_{k}$ be a sequence of analytic subsets of $\Omega$ such that for all $k \in \mathbb{N}, X_{k}$ is of pure dimension $d_{k}$ and $\operatorname{Codim}\left(X_{k}\right)=n-d_{k} \geqslant n-p$. If $\left(C_{k}\right)_{k}$ is a given sequence of strictly positive numbers, then we would like to know the possibility to find a positive and closed current $\Theta$, of bidimension $(p, p)$, globally defined on $\Omega$, such that the generic Lelong number $m_{X_{k}}$ of $\Theta$ along each $X_{k}$ satisfies $m_{X_{k}}=C_{k}$ ?

For convenience, to solve this problem we take essentially the situation where the sequence $\left(\operatorname{mes}\left(X_{k} \cap L\right)\right)_{k}$ is lower bounded for any compact subset $L$ with empty interior in $\Omega$.

Indeed, if a given analytic subset $X_{k}$ intersects some compact $L$, then the area of its intersection with another compact $L^{\prime}$ contained in the interior of $L$ can not has a zero limit.

Furthermore, if for any compact subset $L$ of $\Omega$ we have $\lim _{k \rightarrow \infty} \operatorname{mes}\left(X_{k} \cap L\right)=0$ then the analytic subsets $\left(X_{k}\right)_{k}$ escape to the frontier of $\Omega$. So we may suppose that there exists some compact subset $K$ of $\Omega$ and a constant $\gamma_{K}>0$ such that

$$
\operatorname{mes}\left(X_{k} \cap K\right) \geqslant \gamma_{K}, \quad \text { for all } k \in I,
$$

where $I \subset \mathbb{N}$ is an infinite subset of positive natural numbers. Hence, the sequence $\left(C_{k}\right)_{k \in I}$ will have to be convergent to 0 .

On the other hand, the presented problem has a solution, as well as the sequence $\left(X_{k}\right)_{k}$ satisfies, for any open ball $B(a, r) \subset \Omega$, the following growth condition

$$
\sum_{k} C_{k} \operatorname{mes}\left(X_{k} \cap B(a, r)\right)=\sum_{k \in \mathbb{N}} C_{k} \int_{B(a, r)}\left[X_{k}\right] \wedge \beta^{d_{k}}<\infty .
$$

Eventually, The founded solution $\Theta$ will satisfy for all $k \in \mathbb{N}, C_{k}=m_{X_{k}}$.

We may note that Theorem 0.1 holds true if the codimension $d_{k}$ of each analytic subset $X_{k}$, is taken such that $d_{k}=p$. Indeed, in this case, we may take

$$
\Theta=\sum_{k \in \mathbb{N}} C_{k}\left[X_{k}\right]
$$

The main tools of the proof of Theorem 0.1, will be based on a beautiful result due to Ben Messaoud [1] about the intermediate currents associated to a given positive closed current. Such a result improved an anterior result due to H. Skoda [2] and P. Lelong [3] which is the basis of the proof of Theorem 0.2 .

The originality of our constructions that will appear in the proofs of the main theorems of this paper, lies in the fact that both results of [1] and [2] can be adapted and applied to each $\left(n-d_{k}, n-d_{k}\right)$-current of integration over $X_{k}, k \in \mathbb{N}$. The methods of [1] and [2] will offer practical approaches to construct the current $\Theta$ of Theorem 0.1 and the function $v$ of Theorem 0.2.

The results of [1] and [2] are expressed as follows: 
Theorem (H. Ben Messaoud [1]). Let $T$ be a closed positive $(n-p, n-p)$-current on an open set $\Omega$ in $\mathbb{C}^{n}$. Then for any $\varepsilon>0$ and any natural number $1 \leqslant l \leqslant n-p$, there exists a closed positive $(l, l)$-current $T_{l}$ such that

1. For any point $z \in B(0, r)$, we have $\nu\left(T_{l}, z\right)=\nu(T, z)$.

2. $\nu\left(T_{l}, r\right) \leqslant A \nu(T, r+\varepsilon r) \log ^{2 l} r$, where $A=A(\varepsilon)>0$ is a positive constant.

Theorem (H. Skoda [2]). Let $T$ be a closed positive $(n-p, n-p)$-current on an open set $\Omega$ in $\mathbb{C}^{n}$. Then for any $\varepsilon>0$, there exists a plurisubharmonic function $V$ on $\mathbb{C}^{n}$ such that for any point $z \in B(0, r)$ :

1. $\nu\left(\frac{i}{2 \pi} \partial \bar{\partial} V, z\right)=\nu(T, z)$.

2. $V(z) \leqslant A \nu(T, r+\varepsilon r) \log ^{2} r$, where $A=A(\varepsilon)>0$ is a positive constant.

This paper is organized as follows:

- in Section 2: some preliminaries;

- in Section 3: the proof of Theorem 0.1;

- in Section 4: the proof of Theorem 0.2.

\section{Preliminaries}

In this section we shall present some basic notions needed for the rest of this paper. For more informations related to differential geometry and pluripotential theory, we left the reader to consulte for examples $[1,4,5]$. We take $\Omega$ such that $\Delta^{n} \Subset \Omega$, where $\Delta^{n}$ is the polydisc in $\mathbb{C}^{n}$. For integers $p$ and $q$ such that $1 \leqslant p, q \leqslant n$, we denote $\mathscr{D}_{(p, q)}(\Omega)$ the space of smooth compactly supported-differential forms of bidegree $(p, q)$ on $\Omega$. The dual $\mathscr{D}_{(p, q)}^{\prime}(\Omega)$ is the space of currents of bidimension $(p, q)$ or of bidegree $(n-p, n-q)$ on $\Omega$. A current $T$ of bidimension $(p, p)$ on $\Omega$, is said to be positive if for all $\gamma_{1}, \ldots, \gamma_{p}$ in $\mathscr{D}_{(1,0)}(\Omega)$, the distribution

$$
T \wedge i \gamma_{1} \wedge \bar{\gamma}_{1} \wedge \cdots \wedge i \gamma_{p} \wedge \bar{\gamma}_{p}
$$

is a positive measure. The current $T$ is said to be closed if $d T=0$ where $d=\partial+\bar{\partial}, d^{c}=\frac{i}{2}(\bar{\partial}-\partial)$ and $d d^{c}=i \partial \bar{\partial}$. We denote respectively $\beta(z)=d d^{c}|z|^{2}$ the Kähler form on $\mathbb{C}^{n}$ and $\alpha=d d^{c} \log |z|$. Following [3] (see also [4]), a well known fact that the coefficients $\Theta_{I, J}$ of every positive current $\Theta=\sum_{|I|=|J|=n-p} \Theta_{I, J} d z_{I} \wedge d \bar{z}_{J}$ are complex measures and satisfy $\Theta_{I, J}=\bar{\Theta}_{J, I}$ for multi-indices $|I|=|J|=n-p$. Moreover, the diagonal coefficients $\Theta_{I, I}$ are positive measures and the absolute values $\left|\Theta_{I, J}\right|$ of the measures $\Theta_{I, J}$ are such that

$$
\lambda_{I} \lambda_{J}\left|\Theta_{I, J}\right| \leqslant 2^{p} \sum_{M} \lambda_{M}^{2} \Theta_{I, I}, \quad I \cap J \subset M \subset I \cup J
$$

where $\lambda_{k}$ are arbitrary positive coefficients and $\lambda_{I}=\prod_{\lambda \in I} \lambda_{k}$.

The positive measure $\sigma_{\Theta}=\Theta \wedge \frac{\beta^{p}}{p !}$ is called the trace measure of $\Theta$ and satisfies the inequality

$$
\sigma_{\Theta} \leqslant C\|\Theta\|
$$


where $\|\Theta\|=\sum_{I, J}\left|\Theta_{I, J}\right|$ is the mass measure of $\Theta$ and $C$ is a positive constant.

The limit, as $r \rightarrow 0$, of the ratio

$$
\nu_{\Theta}(z, r)=\frac{\sigma_{\Theta}(B(z, r))}{\frac{\pi^{p}}{p !} r^{2 p}}=\frac{1}{\frac{\pi^{p}}{p !} r^{2 p}} \int_{B(z, r)} \Theta \wedge \beta^{p}
$$

is called the Lelong number of $\Theta$ at point $z$ and is denoted $\nu(\Theta, z)$. At point $z=0$, we put

$$
\nu_{\Theta}(r)=\frac{\sigma_{\Theta}(B(0, r))}{\frac{\pi^{p}}{p !} r^{2 p}} .
$$

For any $c>0$ the upper-level sets for the Lelong numbers of $\Theta$ are denoted by

$$
E_{c}(\Theta)=\{z \in \Omega, \nu(\Theta, z) \geqslant c\} .
$$

According to [5], the sets $E_{c}(\Theta)$ are analytic subsets. Therefore, following [4], $E_{c}(\Theta)$ are closed sets of locally finite $\mathscr{H}_{2 p}$ Hausdorff measure in $\mathbb{C}^{n}$.

Finally, if $A$ is an irreducible analytic subset of $\Omega$, we set

$$
m_{A}=\inf \{\nu(\Theta, z), z \in A\}
$$

and call $m_{A}$ the generic Lelong number of $\Theta$ along $A$.

\section{Proof of Theorem 0.1}

The proof of Theorem 0.1 follows from several steps and will be complete after proving some fondamental propositions. We begin by proceeding locally in a neighborhood of a given point $z_{0} \in \Omega$ such that $r<d\left(z_{0}, C \Omega\right)$ where $r$ is a positive real number. Let $\eta$ be a smooth positive function equal to 1 on $B\left(z_{0}, \frac{r}{2}\right)$ and has a compact support in $B\left(z_{0}, r\right)$. We may assume that $z_{0}=0$. Denote by $h_{k}$ the kernel given by

$$
h_{k}(x)=-\frac{1}{\left(n+d_{k}-p-1\right) \pi^{n+d_{k}-p}}|x|^{-2\left(n+d_{k}-p-1\right)} .
$$

For each current of integration over $X_{k}$ it is associated an $(n-p-1, n-p-1)$ differential form denoted $U_{\eta,\left[X_{k}\right]}$ given by the following integral expression

$$
U_{\eta,\left[X_{k}\right]}(z)=\int_{\xi \in \mathbb{C}^{n}} \eta(\xi) h_{k}(z-\xi) \beta^{n+d_{k}-p-1}(z-\xi) \wedge\left[X_{k}\right](\xi) .
$$

Since the kernel given by (3.3) lies in $L_{l o c}^{1}\left(\mathbb{C}^{n}\right)$, then it is clear that the potential given by (3.4) has locally integrable coefficients in $\mathbb{C}^{n}$. Let $\chi_{k}(x)$ denote the negative current of bidegree $\left(n+d_{k}-p-1, n+d_{k}-p-1\right)$ given by the expression

$$
\chi_{k}(x)=h_{k}(x) \beta^{n+d_{k}-p-1}(x) .
$$

Therefore, the current $\chi_{k}$ defined by (3.5) has locally integrable coefficients.

Proposition 3.1. There are currents respectively denoted $T_{\eta, k}, J_{1}\left(\eta\left[X_{k}\right]\right), J_{2}\left(\eta\left[X_{k}\right]\right)$ and $J_{3}\left(\eta\left[X_{k}\right]\right)$ such that in the weak sense of currents, we have

$$
\frac{i}{2} \partial \bar{\partial} U_{\eta,\left[X_{k}\right]}=T_{\eta, k}+J_{1}\left(\eta\left[X_{k}\right]\right)+J_{2}\left(\eta\left[X_{k}\right]\right)+J_{3}\left(\eta\left[X_{k}\right]\right) .
$$


Proof. Consider the mappings defined by $p_{1}:(x, z) \mapsto x$ and $p_{2}:(x, z) \mapsto z$, they are respectively the first and the second projections from $\mathbb{C}^{n} \times \mathbb{C}^{n}$ to $\mathbb{C}^{n}$. Let $\tau$ denote the mapping from $\mathbb{C}^{n} \times \mathbb{C}^{n}$ to $\mathbb{C}^{n}$ defined by $\tau(x, z)=z-x$. The current $U_{\eta,\left[X_{k}\right]}$ can be expressed as a direct image on $\mathbb{C}^{n} \times \mathbb{C}^{n}$ as the following

$$
U_{\eta,\left[X_{k}\right]}(z)=p_{2 *}\left(\left(\tau^{*} \chi_{k}\right) \wedge p_{1}^{*}\left(\eta\left[X_{k}\right]\right)\right)
$$

The representation given by (3.6) makes the computation of $\frac{i}{2} \partial \bar{\partial} U_{\eta,\left[X_{k}\right]}$, in the weak sense of current, easy and gives

$$
\begin{aligned}
\frac{i}{2} \partial \bar{\partial} U_{\eta,\left[X_{k}\right]} & =\underbrace{p_{2 *}\left(\tau^{*}\left(\frac{i}{2} \partial \bar{\partial} \chi_{k}\right) \wedge p_{1}^{*}\left(\eta\left[X_{k}\right]\right)\right)}_{T_{\eta, k}}+\underbrace{p_{2 *}\left(\tau^{*}\left(\frac{i}{2} \partial \chi_{k}\right) \wedge p_{1}^{*}\left(\bar{\partial} \eta \wedge\left[X_{k}\right]\right)\right)}_{J_{2}\left(\eta\left[X_{k}\right]\right)}- \\
& -\underbrace{p_{2 *}\left(\tau^{*}\left(\frac{i}{2} \bar{\partial} \chi_{k}\right) \wedge p_{1}^{*}\left(\partial \eta \wedge\left[X_{k}\right]\right)\right)}_{J_{3}\left(\eta\left[X_{k}\right]\right)}+\underbrace{p_{2}}_{\underbrace{}_{2 *}\left(\tau^{*} \chi_{k} \wedge p_{1}^{*}\left(\frac{i}{2} \partial \bar{\partial} \eta \wedge\left[X_{k}\right]\right)\right)} .
\end{aligned}
$$

Proposition 3.2. The current $J_{1}\left(\eta\left[X_{k}\right]\right)$ has an integral expression as follows

$$
J_{1}\left(\eta\left[X_{k}\right]\right)=\int_{x \in \mathbb{C}^{n}} K_{1}(x, z) \wedge \partial \eta(x) \wedge\left[X_{k}\right](x) .
$$

The currents $J_{2}\left(\eta\left[X_{k}\right]\right)$ and $J_{3}\left(\eta\left[X_{k}\right]\right)$ have similar representations.

Proof. If $K_{1}(x, z)$ denote the component of bidegree $(n-p, n-p)$ in $z$ and $\left(d_{k}, d_{k}-1\right)$ in $x$ of the form $\tau^{*}\left(\frac{i}{2} \partial \chi_{k}\right)$, then we get

$$
J_{1}\left(\eta\left[X_{k}\right]\right)=\int_{x \in \mathbb{C}^{n}} K_{1}(x, z) \wedge \partial \eta(x) \wedge\left[X_{k}\right](x) .
$$

If $K_{2}(x, z)$ denote the component of bidegree $(n-p, n-p)$ in $z$ and $\left(d_{k}-1, d_{k}\right)$ in $x$ of the form $\tau^{*}\left(\frac{i}{2} \partial \chi_{k}\right)$, then we get

$$
J_{2}\left(\eta\left[X_{k}\right]\right)=\int_{x \in \mathbb{C}^{n}} K_{2}(x, z) \wedge \bar{\partial} \eta(x) \wedge\left[X_{k}\right](x) .
$$

Finally, if $K_{3}(x, z)$ denote the component of bidegree $(n-p, n-p)$ in $z$ and $\left(d_{k}-1, d_{k}-1\right)$ in $x$ of the form $\tau^{*}\left(\frac{i}{2} \partial \chi_{k}\right)$, then we get

$$
J_{3}\left(\eta\left[X_{k}\right]\right)=\int_{x \in \mathbb{C}^{n}} K_{3}(x, z) \wedge \partial \bar{\partial} \eta(x) \wedge\left[X_{k}\right](x) .
$$

Proposition 3.3. The sum of the modulus of the coefficients of the form $J_{1}(z)+J_{2}(z)+J_{3}(z)$, denoted $\left\|J_{1}(z)+J_{2}(z)+J_{3}(z)\right\|$, is such that

$$
\left\|J_{1}(z)+J_{2}(z)+J_{3}(z)\right\| \leqslant A \int_{\xi \in \mathbb{C}^{n}}\left(\frac{\|\partial \eta\|+\|\bar{\partial} \eta\|}{|z-\xi|}+\|\partial \bar{\partial} \eta\|\right)\left|h_{k}\right|(z-\xi) d \sigma_{\left[X_{k}\right]}(\xi)
$$

where $A=A(n, p)$ is a strictly positive constante. 
Proof. Since $\frac{i}{2} \partial \chi_{k}=\sum_{j=1}^{n}|x|^{-2\left(n-p+d_{k}\right)} \bar{x}_{j} d x_{j} \wedge \beta^{n-p+d_{k}-1}$ then we get

$$
\tau^{*}\left(\frac{i}{2} \partial \chi_{k}\right)=\sum_{j=1}^{n}|z-x|^{-2\left(n-p+d_{k}\right)}\left(\bar{z}_{j}-\bar{x}_{j}\right)\left(d z_{j}-d x_{j}\right) \wedge \tau^{*} \beta^{n-p+d_{k}-1} .
$$

In addition, by (2.2), the measures coefficients of the current $\left[X_{k}\right]$ are dominated by the trace measure $\sigma_{\left[X_{k}\right]}$. So the representation (3.7) implies the following estimate

$$
\left\|J_{1}(z)\right\| \leqslant A_{1}(n, p) \int_{\mathbb{C}^{n}}|z-x|^{-2\left(n-p+d_{k}\right)+1}|| \partial \eta(x) \| d \sigma_{\left[X_{k}\right]}(x)
$$

where $\left\|J_{1}(z)\right\|$ is the sum of the modulus of the coefficients of the forme $J_{1}\left(\eta\left[X_{k}\right]\right)$ and $\|\bar{\partial} \eta(x)\|$ is the sum of the modulus of the coefficients of $\bar{\partial} \eta(x)$. Similar procedures for $J_{2}\left(\eta\left[X_{k}\right]\right)$ and $J_{3}\left(\eta\left[X_{k}\right]\right)$ given by representations (3.8) and (3.9) yield similar estimates as in (3.12). These estimates provide the following

$$
\left\|J_{1}(z)+J_{2}(z)+J_{3}(z)\right\| \leqslant A_{2} \int_{\xi \in \mathbb{C}^{n}}\left(\frac{\|\partial \eta\|+\|\bar{\partial} \eta\|}{|z-\xi|}+\|\partial \bar{\partial} \eta\|\right)\left|h_{k}\right|(z-\xi) d \sigma_{\left[X_{k}\right]}(\xi)
$$

where $A_{2}=A_{2}(n, p)$ is a positive constant. The estimate (3.13) allows to measure the default of positivity of the current $\frac{i}{2} \partial \bar{\partial} U_{\eta,\left[X_{k}\right]}$.

Proposition 3.4. The positive current $T_{\eta, k}$ is such that

$$
T_{\eta, k}=p_{2 *}\left(\tau^{*}\left(\alpha^{n+d_{k}-p}\right) \wedge p_{1}^{*}\left(\eta\left[X_{k}\right]\right)\right) .
$$

Furthermore, up to a positive constant, $T_{\eta, k}$ has the same Lelong numbers as the current $\eta\left[X_{k}\right]$.

Proof. To prove the equality (3.14), it suffices to observe that $\frac{i}{2} \partial \bar{\partial} \chi_{k}$ is a closed positive $\left(n+d_{k}-p, n+d_{k}-p\right)$-form on $\mathbb{C}^{n} \backslash\{0\}$ which is invariant under the action of the unitary group $U(n, \mathbb{C})$, then following [4], in the weak sense of currents, we get

$$
\tau^{*}\left(\frac{i}{2} \partial \bar{\partial} \chi_{k}\right)=\tau^{*}\left(\alpha^{n+d_{k}-p}\right)
$$

where $\tau^{*}\left(\frac{i}{2} \partial \chi_{k}\right)$ is given by (3.11). For the second assertion (equality of the Lelong numbers), a detailed proof of this fact was given in [1]. Notice that the current $T_{\eta, k}$ didn't need to be closed. However, if we assume that $T_{\eta, k}$ is closed, we propose the following proof. We may prove the equality of the Lelong numbers at point $z=0$. Since $T_{\eta, k}$ is assumed to be closed, according to [4], we have

$$
\frac{1}{r^{2 p}} \int_{|z|<r} T_{\eta, k} \wedge \beta^{p}=\int_{|z|<r} T_{\eta, k} \wedge \alpha^{p} .
$$

On the other hand, the equality (3.14) means that

$$
T_{\eta, k}(z)=\int_{x \in \mathbb{C}^{n}} \alpha^{n+d_{k}-p}(z-x) \wedge \eta(x)\left[X_{k}\right](x) .
$$

Then, if we put $I(r)=\int_{|z|<r} T_{\eta, k} \wedge \alpha^{p}$, then the equality (3.15) will be as follows

$$
I=\int_{(x, z) \in \mathbb{C}^{n} \times\{|z|<r\}} \alpha^{n+d_{k}-p}(z-x) \wedge \eta(x)\left[X_{k}\right](x) \wedge \alpha^{p}(z) .
$$


By the change of variables $\left(x^{\prime}, z^{\prime}\right)=(x, z-x)$, the equality (3.16) can be written as

$$
I(r)=\int_{\left\{\left(x^{\prime}, z^{\prime}\right) \in \mathbb{C}^{n} \times \mathbb{C}^{n},\left|z^{\prime}-x^{\prime}\right|<r\right\}} \alpha^{n+d_{k}-p}\left(z^{\prime}\right) \wedge \eta\left(x^{\prime}\right)\left[X_{k}\right]\left(x^{\prime}\right) \wedge \alpha^{p}\left(z^{\prime}-x^{\prime}\right) .
$$

By the Fubini theorem and the change of variables $\xi=x^{\prime}-z^{\prime}$, the equality (3.17) can be transformed as

$$
\begin{aligned}
I(r) & =\int_{x^{\prime} \in \mathbb{C}^{n}}\left[\int_{\left|z^{\prime}-x^{\prime}\right|<r} \eta\left(x^{\prime}-z^{\prime}\right)\left[X_{k}\right]\left(x^{\prime}-z^{\prime}\right) \wedge \alpha^{n+d_{k}-p}\left(z^{\prime}\right) \wedge \alpha^{p}\left(z^{\prime}-x^{\prime}\right)\right]= \\
& =\int_{x^{\prime} \in \mathbb{C}^{n}}\left[\int_{|\xi|<r} \eta(\xi)\left[X_{k}\right](\xi) \wedge \alpha^{n+d_{k}-p}\left(x^{\prime}-\xi\right) \wedge \alpha^{p}(\xi)\right] .
\end{aligned}
$$

Since only components of bidegree $\left(d_{k}, d_{k}\right)$ in $\xi$ and of bidegree $(n-p, n-p)$ in $x^{\prime}$ of the form $\alpha^{n+d_{k}-p}\left(x^{\prime}-\xi\right) \wedge \alpha^{p}(\xi)$ are useful and since by [4], $\int_{x^{\prime} \in \mathbb{C}^{n}} \alpha^{n}\left(x^{\prime}\right)=1$ because $\int_{|\xi-a|<r}\left(d d^{c} \log |\xi-a|\right)^{n}=1$ for all $r>0$ and all $a \in \mathbb{C}^{n}$. Then, by letting $r \rightarrow 0$ in (3.18), we find that $\nu\left(T_{\eta, k}, 0\right)=C \nu\left(\eta\left[X_{k}\right], 0\right)$ where $C=C(n, p)$ is a positive constant.

From now, we will proceed globally.

Proposition 3.5. For all $k \in \mathbb{N}$, there exist global currents respectively denoted $U_{\left[X_{k}\right]}, T_{k}, \Phi_{1, k}, \Phi_{2, k}, \Phi_{3, k}, \Phi_{4, k}$ such that $\frac{i}{2} \partial \bar{\partial} U_{\left[X_{k}\right]}$ is decomposed into

$$
\frac{i}{2} \partial \bar{\partial} U_{\left[X_{k}\right]}=T_{k}+\Phi_{1, k}+\Phi_{2, k}+\Phi_{3, k}+\Phi_{4, k} .
$$

Proof. There exists an open cover $\left(\Omega_{j}\right)_{j}$ for $\Omega$, by relatively compact open subsets such that $\Omega_{j} \subset \Omega_{j+1}$ and $\Omega=\bigcup_{j} \Omega_{j}$. It is clear that $\left(\Omega_{j}^{\prime}\right)_{j}$ such that $\Omega_{j}^{\prime}=\Omega_{j+1} \backslash \bar{\Omega}_{j}$, is a locally finite open cover for $\Omega$ that is subordinate to $\left(\Omega_{j}\right)_{j}$. Let $\left(\omega_{j}\right)_{j}$ such that $\omega_{j} \Subset \Omega_{j+1} \backslash \bar{\Omega}_{j}$ and $\left(\omega_{j}\right)_{j}$ still cover $\Omega$. Consider $\left\{\left(\rho_{j}, \Omega_{j}^{\prime}\right), j \in \mathbb{N}\right\}$ a partition of unity on $\Omega$ such that $\operatorname{Supp} \rho_{j} \subset \Omega_{j}^{\prime}$ and $\rho_{j}=1$ on $\bar{\omega}_{j}$. By sticking the currents $U_{\eta_{j},\left[X_{k}\right]}$, we can now construct a global potential defined by

$$
U_{\left[X_{k}\right]}(z)=\sum_{j=1}^{\infty} \rho_{j}(z) U_{\eta_{j},\left[X_{k}\right]}(z), \quad k \in \mathbb{N}
$$

An easy computation, using equality (3.19), in the weak sens of currents, yields

$$
\begin{aligned}
\frac{i}{2} \partial \bar{\partial} U_{\left[X_{k}\right]} & =\sum_{j} \rho_{j} \frac{i}{2} \partial \bar{\partial} U_{\eta_{j},\left[X_{k}\right]}+ \\
& +\sum_{j} \frac{i}{2} \partial \rho_{j} \wedge \bar{\partial} U_{\eta_{j},\left[X_{k}\right]}- \\
& -\sum_{j} \frac{i}{2} \bar{\partial} \rho_{j} \wedge \partial U_{\eta_{j},\left[X_{k}\right]}+ \\
& +\sum_{j} \frac{i}{2} \partial \bar{\partial} \rho_{j} \wedge U_{\eta_{j},\left[X_{k}\right]}
\end{aligned}
$$

Since $\frac{i}{2} \partial \bar{\partial} U_{\eta_{j},\left[X_{k}\right]}=T_{\eta_{j}, k}+J_{1}\left(\eta_{j}\left[X_{k}\right]\right)+J_{2}\left(\eta_{j}\left[X_{k}\right]\right)+J_{3}\left(\eta_{j}\left[X_{k}\right]\right)$, then by taking in account 
equality (3.20), the current $\frac{i}{2} \partial \bar{\partial} U_{\left[X_{k}\right]}$ can be decomposed into

$$
\begin{aligned}
\frac{i}{2} \partial \bar{\partial} U_{\left[X_{k}\right]} & =\underbrace{\sum_{j} \rho_{j} T_{\eta_{j}, k}}_{T_{k}}+\underbrace{\sum_{j} \rho_{j}\left(J_{1}\left(\eta_{j}\left[X_{k}\right]\right)+J_{2}\left(\eta_{j}\left[X_{k}\right]\right)+J_{3}\left(\eta_{j}\left[X_{k}\right]\right)\right)}_{\Phi_{1, k}}+ \\
& +\underbrace{\sum_{j} \frac{i}{2} \partial \rho_{j} \wedge \bar{\partial} U_{\eta_{j},\left[X_{k}\right]}}_{\Phi_{2, k}}-\underbrace{\sum_{j} \frac{i}{2} \bar{\partial} \rho_{j} \wedge \partial U_{\eta_{j},\left[X_{k}\right]}}_{\Phi_{3, k}}+\underbrace{\sum_{j} \frac{i}{2} \partial \bar{\partial} \rho_{j} \wedge U_{\eta_{j},\left[X_{k}\right]}}_{\Phi_{4, k}} .
\end{aligned}
$$

Proposition 3.6. For all $k$, there exists a positive closed current $\Theta_{k}$ of bidimension $(p, p)$ on $\Omega$, such that for any $\varepsilon>0$ and any open ball $B(a, r) \Subset \Omega$, we have

$$
\sigma_{\Theta_{k}}(B(a, r)) \leqslant A_{3}(\varepsilon, r, n, p) \sigma_{\left[X_{k}\right]}(B(a, r+\varepsilon r))
$$

where $A_{3}=A_{3}(\varepsilon, r, n, p)$ is a positive constant.

Proof. We may choice the functions $\left(\eta_{j}\right)_{j}$ such that for all $j, \operatorname{Supp}\left(\eta_{j}\right) \subset \Omega_{j}$. This makes that each form $\Phi_{s, k}, s \in\{1,2,3,4\}$, is smooth in a neighborhood of $\operatorname{Supp}\left(\rho_{j}\right)$. Furthermore, following [1], for any $\varepsilon>0$ and any point $z \in \operatorname{Supp}\left(\rho_{j}\right)$, the sum of the modulus of the coefficients of the form

$$
\Phi_{k}(z)=\Phi_{1, k}(z)+\Phi_{2, k}(z)+\Phi_{3, k}(z)+\Phi_{4, k}(z),
$$

denoted $\left\|\Phi_{k}(z)\right\|$, satisfies the following estimate

$$
\left\|\Phi_{k}(z)\right\| \leqslant A_{4}(1+|z|)^{-2(n-p)} \nu_{\left[X_{k}\right]}((1+5 \varepsilon)(1+|z|))
$$

where $A_{4}=A_{4}(\varepsilon, n, p)$ is a positive constant. The estimate (3.21) is a default of positivity of the current $\frac{i}{2} \partial \bar{\partial} U_{\left[X_{k}\right]}$. By adding to $\frac{i}{2} \partial \bar{\partial} U_{\left[X_{k}\right]}$ a smooth and closed form sufficiently positive and of course having zero Lelong number every where, of the form $\left(\frac{i}{2} \partial \bar{\partial} w_{k}\right)^{n-p}$ where $w_{k}$ is a smooth strictly plurisubharmonic function (see [6]), the potential $U_{\left[X_{k}\right]}$ provides an $(n-p, n-p)$-positive current defined by

$$
\Theta_{k}=\frac{i}{2} \partial \bar{\partial} U_{\left[X_{k}\right]}+\left(\frac{i}{2} \partial \bar{\partial} w_{k}\right)^{n-p}=T_{k}+\Phi_{k}+\left(\frac{i}{2} \partial \bar{\partial} w_{k}\right)^{n-p} .
$$

In addition, following [1], the current $\Theta_{k}$ given by (3.22), satisfies for any $\varepsilon>0$ and any open ball $B(a, r) \Subset \Omega$

$$
\nu_{\Theta_{k}}(a, r) \leqslant A_{5}(\varepsilon)(\log r)^{2(n-p)} \nu_{\left[X_{k}\right]}(a, r+\varepsilon r) .
$$

Consequently, (3.22) provides the following

$$
\sigma_{\Theta_{k}}(B(a, r)) \leqslant A_{6}(\varepsilon) \frac{\pi^{p-d_{k}} d_{k} !}{(1+\varepsilon)^{2 d_{k}} p !}(\log r)^{2(n-p)} r^{2\left(p-d_{k}\right)} \sigma_{\left[X_{k}\right]}(B(a, r+\varepsilon r)) .
$$

Since for all $k \in \mathbb{N}$, we have $\frac{\pi^{p-d_{k}} d_{k} !}{(1+\varepsilon)^{2 d_{k}} p !} \leqslant \pi^{p}$ and $r^{2\left(p-d_{k}\right)} \leqslant \max \left(1, r^{2 p}\right)$, the estimation (3.24) can be written as

$$
\sigma_{\Theta_{k}}\left((B(a, r)) \leqslant A_{7}(\varepsilon, r, n, p) \sigma_{\left[X_{k}\right]}(B(a, r+\varepsilon r))\right.
$$

where $A_{7}(\varepsilon, r, n, p)=\pi^{p} A_{6}(\varepsilon) \max \left(1, r^{2 p}\right)(\log r)^{2 n-2 p}$.

Proposition 3.7. There exists a closed positive current $\Theta$ of bidimension $(p, p)$ on $\Omega$, such that for all $k \in \mathbb{N}$ and any point $z \in X_{k}$, we have $\nu(\Theta, z) \geqslant C_{k}$. 
Proof. Put $\Gamma_{N}=\sum_{1 \leqslant k \leqslant N} C_{k} \Theta_{k}$. using the estimates (3.23), (3.24), (3.25) and the condition (0.1), the mass of the current $\Gamma_{N}$ over a given ball $B(a, r) \Subset \Omega$, satisfies, for any $\varepsilon>0$, the following estimate

$$
\begin{aligned}
\left\|\Gamma_{N}\right\|_{B(a, r)} & \leqslant \sum_{1 \leqslant k \leqslant N} C_{k} \sigma_{\Theta_{k}}(B(a, r)) \leqslant \\
& \leqslant A_{7}(\varepsilon, r, n, p) \sum_{k} C_{k} \sigma_{\left[X_{k}\right]}(B(a, r+\varepsilon r))< \\
& <\infty .
\end{aligned}
$$

Therefore, $\left(\Gamma_{N}\right)_{N}$ is an increasing sequence of positive closed currents. Further, it is locally bounded in mass independently of $N$. Let $\Theta$ denote its weak limit. Following Proposition 3.4, for any $k \in \mathbb{N}$ and any point $z \in X_{k}$ we have

$$
\nu\left(\Theta_{k}, z\right)=\nu\left(\left[X_{k}\right], z\right),
$$

then according to [4], we may conclude that, for all $1 \leqslant k \leqslant N$ and any point $z \in X_{k}$, we have

$$
\nu(\Theta, z) \geqslant \limsup _{N \rightarrow \infty} \nu\left(\Gamma_{N}, z\right) \geqslant \limsup _{N \rightarrow \infty} \sum_{1 \leqslant j \leqslant N} C_{j} \nu\left(\left[X_{j}\right], z\right) \geqslant C_{k} .
$$

The proof is achieved.

\section{Proof of Theorem 0.2}

There are tow mains steps. The first consists of the construction, for all $k \in \mathbb{N}$, of a plurisubhrmonic function $\tilde{v}_{k}$ on $\Omega$, satisfying on every open ball $B(a, r) \Subset \Omega$, the following statements as in [2].

$$
\begin{gathered}
\nu\left(d d^{c} \tilde{v}_{k}, z\right)=\nu\left(\left[X_{k}\right], z\right) . \\
\forall \varepsilon>0 \text { (small enough), } \quad \tilde{v}_{k}(z) \leqslant A(\varepsilon) \nu\left(\left[X_{k}\right],(1+\varepsilon) r\right) \log ^{2} r,
\end{gathered}
$$

where $A(\varepsilon)$ is a positive constant. To do this, we consider a locally finite open covering $\left(\omega_{j}\right)$ of $\Omega$ by relatively compact open balls contained in a coordinate patches of $\Omega$. We choose concentric balls $\omega_{j}^{\prime \prime} \subset \omega_{j}^{\prime} \subset \omega_{j}$ of respective radii $\frac{r}{3}, \frac{2 r}{3}, r$ such that $\omega_{j}^{\prime \prime}$ still cover $\Omega$. According to [2], for all $\varepsilon>0$, there exists a plurisubharmonic function $v_{k, j}$ on $\Omega$ such that, analogous to the statements (4.26) and (4.27), hold for any point $z \in \omega_{j}^{\prime \prime}$. By a slight modification on $\omega_{j} \backslash \omega_{j}^{\prime}$, we may replace the function $v_{k, j}$ by the function

$$
\tilde{v}_{k, j}=\left\{\begin{array}{lll}
\max \left(v_{k, j}, A(\varepsilon) \nu\left(\left[X_{k}\right],(1+\varepsilon) \frac{r}{3}\right) \log ^{2} \frac{r}{3}\right), & \text { on } \omega_{j} \backslash \omega_{j}^{\prime} \\
v_{k, j}, & \text { on } \omega_{j}^{\prime} .
\end{array}\right.
$$

This modification ensure that statement (4.26) holds for $\tilde{v}_{k, j}$ on $\omega_{j}^{\prime \prime}$, and up to a positive constant, statement (4.27) holds for $\tilde{v}_{k, j}$ on $\omega_{j}$. Let consider now a partition of unity on $\Omega,\left\{\left(\rho_{j}, \omega_{j}\right), j \in \mathbb{N}\right\}$, such that $\operatorname{Supp} \rho_{j} \subset \omega_{j}^{\prime}$ and $\rho_{j}=1$ on $\bar{\omega}_{j}^{\prime \prime}$. Define $\tilde{v}_{k}=\sum_{j} \rho_{j} \tilde{v}_{k, j}$, then the function $\tilde{v}_{k}$ is plurisubharmonic on $\Omega$ since $\rho_{j}=1$ on $\bar{\omega}_{j}^{\prime \prime}$ and $\omega_{j}^{\prime \prime}$ still cover $\Omega$. In addition, by definition of the function $\tilde{v}_{k, j}$ on $\omega_{j} \backslash \omega_{j}^{\prime}$, we have $\tilde{v}_{k} \not \equiv-\infty$. It also satisfies statements (4.26) and (4.27) on any small open ball $B\left(a, r_{0}\right)$ contained in some open ball of the form $\omega_{j_{0}}^{\prime \prime}$. Moreover, for all $k \in \mathbb{N}$, 
the mass of $d d^{c} \tilde{v}_{k}$ over $B\left(a, r_{0}\right)$, satisfies the following estimate

$$
\begin{aligned}
\left\|d d^{c} \tilde{v}_{k}\right\|_{B\left(a, r_{0}\right)} & \leqslant A_{1} \int_{\omega_{j_{0}}^{\prime \prime}} d d^{c} \tilde{v}_{k, j_{0}} \wedge \beta^{n-1} \leqslant \\
& \leqslant A_{2} \int_{\omega_{j_{0}}^{\prime}} d d^{c} \rho_{j_{0}} \wedge \tilde{v}_{k, j_{0}} \wedge \beta^{n-1} \leqslant \\
& \leqslant A_{3} \int_{\omega_{j_{0}}} d d^{c} \rho_{j_{0}} \wedge \tilde{v}_{k, j_{0}} \wedge \beta^{n-1} \leqslant \\
& \leqslant A_{4} \nu\left(\left[X_{k}\right],(1+\varepsilon) r_{0}\right) \log ^{2} r_{0} \leqslant \\
& \leqslant A_{5} \operatorname{mes}\left(X_{k} \cap B\left(a, 2 r_{0}\right)\right),
\end{aligned}
$$

where $A_{s}=A_{s}(\epsilon, r), s=1,2,3,4,5$ are positive constants.

The second step consists of considering the sequence of plurisubharmonic functions on $\Omega$ defined by $\tilde{V}_{N}=\sum_{k=1}^{N} C_{k} \tilde{v}_{k}$. It is clear by construction that for all $N \in \mathbb{N}, \tilde{V}_{N} \not \equiv-\infty$. For such a sequence, we may find a contant $M$ such that $\left(\tilde{V}_{N}-M\right)_{N}$ decreases pointwise. Further, following (4.28), for all $N \in \mathbb{N}$, the mass of $d d^{c} \tilde{V}_{N}$, over any open ball $B\left(a, r_{0}\right) \subset \Omega$, satisfies the following estimate

$$
\left\|d d^{c} \tilde{V}_{N}\right\|_{B\left(a, r_{0}\right)} \leqslant A_{5}(\varepsilon, r) \sum_{k} C_{k} \operatorname{mes}\left(X_{k} \cap B\left(a, 2 r_{0}\right)\right)<\infty
$$

where $A_{5}(\varepsilon, r)$ is a positive constant. The estimate $(4.29)$ implies that $\left(d d^{c} \tilde{V}_{N}\right)_{N}$ is an increasing sequence of $(1,1)$ closed positive current having a locally finite mass. Then it has a weak limit as $N \rightarrow \infty$. Put

$$
d d^{c} \tilde{V}=\lim _{N \rightarrow \infty} d d^{c} \tilde{V}_{N}
$$

we have in the weak sens of currents $d d^{c} \tilde{V}=\sum_{k=1}^{\infty} C_{k} d d^{c} \tilde{v}_{k}$. In addition, following (4.26), for any $k \in \mathbb{N}$ and for any point $z \in X_{k}$, we have $\nu\left(d d^{c} \tilde{V}, z\right) \geqslant C_{k}$ which means that for all $k \in \mathbb{N}$, we have $E_{C_{k}}\left(d d^{c} \tilde{V}\right) \supset X_{k}$.

The proof is achieved.

In the following example we apply our main results to polynomial functions.

Example. Take $n=2, p=1, \Omega=\mathbb{C}^{2}$ and denote $\pi$ the projection map $\left(z_{1}, z_{2}\right) \mapsto z_{1}$. Consider the map $F=\left(f_{1}, f_{2}\right)$ such that $F(0)=0$ and $f_{1}, f_{2}$ are polyndmial functions of degree $d$. For any natural number $k \geqslant 2$, we denote respectively $F_{k}$ and $G_{k}$ the composite functions given by

$$
F_{k}=F \circ \cdots \circ F \quad(k \text {-times }) \text { and } G_{k}=\pi \circ F_{k} .
$$

Then $G_{k}$ is a polynomial function of degree $d^{k}$. If $X_{k}=G_{k}^{-1}(0)$, then $\left(X_{k}\right)_{k \in \mathbb{N}^{*}}$ is a sequence of analytic subsets in $\Omega$, such that for all $k, \operatorname{Codim} X_{k} \geqslant 1$. If we choose $C_{k}=d^{-k} \varepsilon_{k}$, where $\left(\varepsilon_{k}\right)_{k}$ is any sequence of strictly positive numbers such that $\sum_{k} \varepsilon_{k}<\infty$, then by Theorem 0.1 , there exists a positive and closed current $\Theta$ of bidimension $(1,1)$ on $\Omega$, such that for all $k \in \mathbb{N}^{*}$, the sublevel sets $E_{C_{k}}(\Theta)$ are such that $E_{C_{k}}(\Theta) \supset X_{k}$. Moreover, by Theorem 0.2 there exists a plurisubharmonic function $v$ on $\Omega$, such that for all $k \in \mathbb{N}$, the sublevel sets $E_{C_{k}}\left(d d^{c} v\right)$, are such that $E_{C_{k}}\left(d d^{c} v\right) \supset X_{k}$.

The author would like to acknowledge the valuable comments and suggestions from the anonymous referees. 


\title{
References
}

[1] H.Ben Messaoud, Intermediate currents associated with a closed positive current, In: Séminaire d'Analyse P.Lelong - P.Dolbeault - H. Skoda, Lecture Notes in Mathematics, Vol. 1028, 1983, 41-68.

[2] H.Skoda, New methods for the study of potentials associated with analytical sets, Séminaire P.Lelong, Lectures Notes in Math, Vol. 410, 1972, 117-141.

[3] P.Lelong, Sur la structure des courants positifs fermés, Séminaire Pierre Lelong, Lecture Notes in Math., Vol. 578, Springer, Berlin, 1977, 136-156.

[4] J.-P.Demailly, Complex Analytic and Differential Geometry, 2012, available at http://wwwfourier.ujf.-grenoble.fr/ demailly/books.html.

[5] Y.-T.Siu, Analyticity of sets associated to Lelong numbers and the extension of closed positive currents, Invent. Math, 27(1974), 53-156.

[6] H.Skoda, Sous ensembles analytiques d'ordre fini ou infini dans $\mathbb{C}^{n}$, Bull. Soc. Math. France, 100(1972), 353-408.

\section{О построении положительных замкнутых потоков с заданными числами Лелона}

Хеди Хедхири

Университет Монастира Монастир, Тунис

\begin{abstract}
Аннотация. Мы устанавливаем, что последовательность $\left(X_{k}\right)_{k \in \mathbb{N}}$ аналитических подмножеств области $\Omega$ в $\mathbb{C}^{n}$, рассчитанная по размеру, может быть выпущена как семейство наборов верхнего уровня для чисел Лелона некоторого положительного замкнутого тока. Это верно тогда, когда последовательность $\left(X_{k}\right)_{k \in \mathbb{N}}$ удовлетворяет для любого компактного подмножества $L$ в $\Omega$, условие роста $\sum_{k \in \mathbb{N}} C_{k} \operatorname{mes}\left(X_{k} \cap L\right)<\infty$. Точнее, мы построили положительный замкнутый ток $\Theta$ двумерности $(p, p)$ на $\Omega$ так, чтобы общее число Лелона $m_{X_{k}}$ из $\Theta$ вдоль каждого $X_{k}$ удовлетворяло $m_{X_{k}}=C_{k}$. В частности, мы доказываем существование плюрисубгармонической функции $v$ на $\Omega$ такой, что каждый $X_{k}$ содержится во множестве верхнего уровня $E_{C_{k}}\left(d d^{c} v\right)$.
\end{abstract}

Ключевые слова: замкнутый положительный ток, плюрисубгармоническая функция, потенциал, аналитическое множество, число Лелона. 